\title{
Network architectures for supporting survivable WDM rings
}

\author{
Aradhana Narula-Tam ${ }^{\dagger}$ \\ MIT Lincoln Laboratory, 244 Wood Street, Lexington, MA 02420 arad@1l.mit.edu \\ Eytan Modiano
}

MIT Laboratory for Information and Decision Systems, Cambridge, MA 02139 modiano@mit.edu

\begin{abstract}
With WDM, a single physical link failure may correspond to multiple logical link failures. We consider the design of physical topologies that ensure logical rings can be embedded in a survivable manner. (C)2002 Optical Society of America

OCIS codes: (060.0060) Fiber optics and optical communications; (060.4250) Networks
\end{abstract}

\section{Introduction}

Wavelength Division Multiplex (WDM) based networks consist of a logical topology, defined by a set of nodes and lightpath connections and a physical topology, defined by the set of nodes and the fiber connecting them. Although both the logical and physical topologies may be independently tolerant to single link failures, once the logical topology is embedded on the physical topology, the logical topology may no longer be survivable to single physical link failures. Each physical fiber link may carry multiple lightpaths. Hence, the failure of a single physical link, can lead to the failure of multiple links in the logical topology which may subsequently leave the logical topology disconnected. Our focus is on the design of physical topologies capable of supporting ring logical topologies in a survivable manner. While there has been a great deal of work in the area of optical layer protection [1,2], this survivable routing formulation is a new approach to network protection that has significant implications on the design of future WDM-based networks $[3,4]$.

In [3] we considered the problem of embedding ring logical topologies on a given physical topology in a manner that ensures the logical topology remains connected in the event of a physical link failure. We call such embeddings "survivable". One of the key results observed in [3] is that for many physical topologies it is not possible to embed ring logical topologies in a survivable manner. For example, nearly $50 \%$ of 9 node rings cannot be embedded, in a survivable manner, in the 11 node NJLATA network shown in Figure 1. Similar results were also obtained for other commonly used physical topologies. Hence, in this paper we focus on the dual problem: How should the physical topology be designed so that it can support logical rings in a survivable manner? In particular, we investigate properties of physical topologies that enable multiple logical rings to be established in a survivable manner and use these properties to design suitable physical topologies.

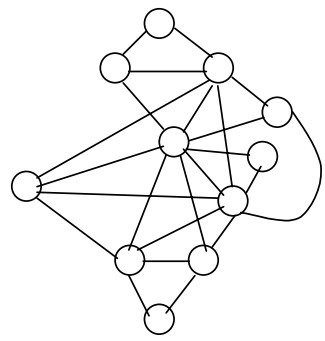

Figure 1. NJ LATA

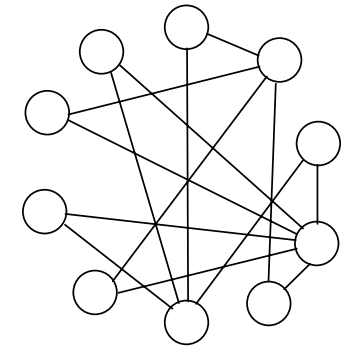

Figure 2. Physical topology generated by ILP for $K=6, R=20$.

We consider the design of $N$ node physical topologies that can support survivable routings of ring logical topologies of size $K \leq N$. Note that a ring of size 3 can be embedded in a survivable manner on any 2-connected physical topology. We thus focus on the problem of embedding rings of size $K \geq 4$. We approach the design problem from two angles. First, we formulate the problem as an Integer Linear Program (ILP) to design the physical topology using the minimum number of physical links that allows a set of random logical topologies to be routed in a survivable manner. Second, we analytically determine necessary conditions on the physical topology for ensuring all $K$ node ring permutations can be embedded in a survivable manner. These conditions lead to lower bound requirements on the number of physical links. Finally, we use the insights gained from the simulation and analytical results to design physical topologies which can support all ring permutations in a survivable manner.

\section{Integer Linear Programming Formulation}

The problem of finding a physical topology with a minimum number of physical links and the associated survivable routings for a batch of $R$ ring logical topologies with $K$ nodes each can be formulated as an ILP. We use Theorem

\footnotetext{
${ }^{\dagger}$ This work is sponsored by DARPA under contract \#F19628-00-C-0002. Opinions, interpretations, recommendations and conclusions are those of the author and are not necessarily endorsed by the Department of Defense.
} 
1 below to determine a survivable routing for each of the $R$ rings, such that each logical topology remains connected even in the event of a physical link failure. In order to route a logical link $(s, t)$ on the physical topology one must find a corresponding path on the physical topology between nodes $s$ and $t$. Such a lightpath consists of a set of physical links connecting nodes $s$ and $t$ as well as wavelengths along those links. Let $f_{i j}^{s t}=1$ if logical link $(s, t)$ is routed on physical link $(i, j)$ and 0 otherwise.

We consider a bi-directional physical topology with nodes $N$ and edges $E$. Similarly, each bi-directional logical topology consists of a set of nodes $N_{L}$ and edges $E_{L}$. A cut is a partition of the set of nodes $N$ into two parts, $S$ and $N-S$. The cut-set corresponds to the set of edges in $E$ that have one endpoint in $S$ and the other in $N-S$. The following theorem, proven in [3], gives necessary and sufficient conditions for survivable routing.

Theorem 1: Let $E(s, t)$ be the set of physical links used by logical link $(s, t)$, i.e., $E(s, t)=\left\{(i, j) \in E\right.$ for which $\left.f_{i j}^{s t}=1\right\}$. A routing is survivable if and only if for every cut-set $\operatorname{CS}\left(S, N_{L}-S\right)$ of the logical topology $\bigcap_{(s, t) \in C\left(S\left(S, N_{L}-S\right)\right.} E$.

The above condition requires that no single physical link is shared by all logical links belonging to a cut-set of the logical topology. In other words, not all of the logical links belonging to a cut-set can be routed on the same physical link. This condition must hold for all cut-sets of the logical topology. For ring logical topologies, this theorem implies that no two logical links can be mapped on the same physical link. Our linear programming algorithm starts with a fully-connected physical topology and assigns a cost of 1 to each physical link that is used. The batch of $R$ rings is embedded simultaneously, and we assign $y_{i j}=1$ if any lightpath uses physical link $(i, j)$.

We can now formulate the physical topology design problem as the following ILP, with the objective of minimizing the total number of physical links used.

Integer Linear Program for finding physical topology for embedding $R$ survivable rings:

Minimize $\sum_{(i, j) \in E} y_{i j}$ subject to:

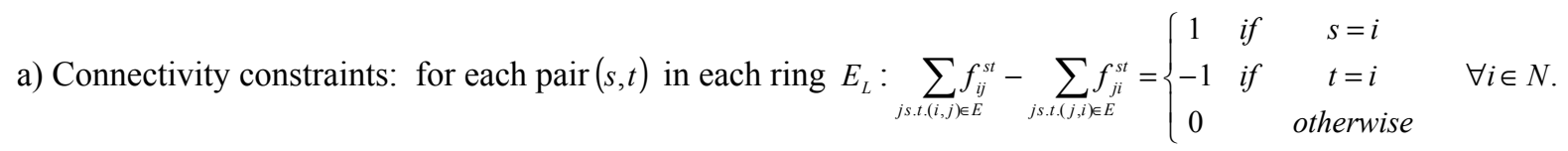

b) Survivability constraints for each logical ring $E_{L}: \sum_{(s, t) \in E_{L}} f_{i j}^{s t}+\sum_{(s, t) \in E_{L}} f_{j i}^{s t} \leq 1, \quad \forall(i, j) \in E$.

c) Physical link use constraints: $y_{i j} \geq f_{i j}^{s t}, \quad \forall(i, j) \in E, \quad \forall(s, t) \in E_{L}, \forall E_{L}$

d) Integer flow constraints: $f_{i j}^{s t} \in\{0,1\}$

This ILP formulation can be made stronger by aggregating the forcing constraints in c) [5]. We have investigated both formulations and found that the solution of the ILP can only be determined for small problem instances, for example, with $N=10, K=6$, and $R=20$, a physical topology solution is found within 24 hours on a SPARC Ultra 10. The resulting physical topology is shown in Figure 2. Due to the complexity of the linear programming solution, this approach cannot be used for general design. However, attributes of the resulting topologies found through experiments provide insights to the physical topology design problem. For example, we find that (1) all nodes in the resulting physical topology have an even degree, (2) the physical topology does not contain a Hamiltonian cycle, and (3) the physical topology has a multi-hub structure.

\section{Necessary conditions for survivable routing}

In addition to the insight gained from the linear programming approach, we develop some necessary conditions on the physical topology to ensure survivable routing of ring logical topologies. Consider any random ring logical topology. For any cut $\langle S, N-S\rangle$ of the physical topology, let $\left|\mathrm{CS}_{\mathrm{p}}(S, N-S)\right|$ be the number of physical links along this cut and $\left|\mathrm{CS}_{\mathrm{L}}(S, N-S)\right|$ be the number of logical links traversing the same cut. Clearly, in order to be able to route the logical links along disjoint physical paths, $\left|\mathrm{CS}_{\mathrm{p}}(S, N-S)\right|$ must be greater than or equal to $\left|\mathrm{CS}_{\mathrm{L}}(S, N-S)\right|$ for each cut. This condition is necessary, but not sufficient to insure that a survivable routing exists for a particular ring logical topology. For embedding all possible $K$ node ring logical topologies in a survivable manner we obtain the following necessary condition on the physical topology.

Theorem 2: For a physical topology to support any possible $K$ node ring logical topology in a survivable manner the following must hold. For any cut of the physical topology $\langle S, N-S\rangle,\left|C S_{p}(S, N-S)\right| \geq 2 \min (|S|,|N-S|,\lfloor K / 2\rfloor)$.

Theorem 2 says that for all cuts of the physical topology, the number of physical links in the cut set must be greater than or equal to twice the minimum of the number of nodes on the smaller side of the cut and $\lfloor K / 2\rfloor$, where 
$\lfloor K / 2\rfloor$ corresponds to the maximum number of nodes in a $K$ node ring logical topology that can be on both sides of the cut. Due to space limitations, we do not prove Theorem 2 here.

By considering all two node cut-sets, we obtain the following corollary as a direct result of Theorem 2.

Corollary 1: Any node of degree 2 must have neighbors of degree 4 or higher.

Proof: Suppose a node of degree 2 has a neighbor of degree 3, then the cut-set consisting of the degree 2 node and its degree 3 neighbor contains only 3 links. However, since the cut-set contains two nodes, Theorem 2 requires a minimum of 4 cut-set links.

In the following section, we use this corollary in the design of effective physical topologies. Corollary 1 and Theorem 2 also lead to the following lower bound on the physical link requirement.

Corollary 2: To support all logical rings of size $K \geq 4$, an $N$ node physical topology must have at least $4 N / 3$ links. The proof of Corollary 2 is omitted in the interest of brevity.

\section{Physical Topologies that Ensure Survivable Ring Routing}

The simulation (ILP) and analytical results provide valuable insights in designing physical topologies that can support ring permutations of various sizes. From Corollary 1, we know that all degree 2 nodes must have neighbors of degree 4 or higher. The physical topologies designed through the ILP simulations all have hub structures, i.e., a small number of nodes with large degree and the remaining nodes with degree 2. Furthermore, all nodes in the generated topologies have even degree. These insights are used to design the following physical topology which can support all ring permutations of size $K \leq N-2$ :

Dual Hub Architecture: Consider a physical topology with $N$ nodes, two of which are hub nodes. Each non-hub node has degree 2 and is connected to both hub nodes. The hub nodes each have degree $N-2$.

An example of the resulting physical topology for $N=10$ nodes is given in Figure 3 . The dual hub architecture contains $2(N-2)$ bi-directional physical links.

Theorem 3: The dual hub architecture can support survivable routings of all logical rings of size $K \leq N-2$.

Proof: To prove that this physical topology can support all rings of size $N-2$, we examine the cases where the logical ring contains 0,1 , or 2 , of the hub nodes. First consider the case where the ring logical topology contains none of the hub nodes. Starting with any node in the logical ring, a path can be found to the next node via one of the hub nodes. The path to the subsequent node will then go through the other hub node. Thus consecutive lightpaths in the logical ring alternate between using each of the two hubs as intermediate nodes. Each hub node can be used as an intermediate node $(N-2) / 2$ times, thus we can support $N-2$ lightpaths. Proofs for the cases where the logical topology contains one or both hub nodes are similar.

Furthermore, we can show that the dual hub architecture is optimal in the following sense:

Theorem 4: The dual hub architecture uses the minimum number of physical links necessary to support all logical rings of size $N-2$ in a survivable manner.

To prove Theorem 4 we show that for any physical topology with fewer than $2(N-2)$ links, we can find an $N-2$ node ring logical topology where each logical link requires two physical links (for a total of $2(N-2)$ links). Hence a physical topology with fewer than $2(N-2)$ links cannot support all $N-2$ node logical topologies. Embedding rings of size $N-1$ or $N$ is considerably more difficult. We have designed an architecture that supports all $N$ node rings, however, it requires order $4 N$ rather than order $2 N$ physical links.

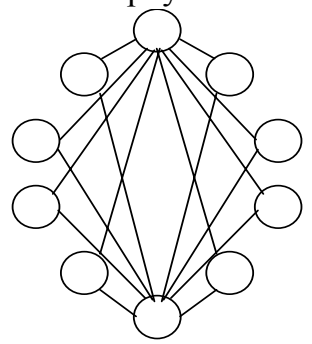

Figure 3: Physical topology that supports all size $N-2$ rings.

References:

[1] S. Ramamuthy and B. Mukherjee, "Survivable WDM mesh networks: Part I - Protection,", IEEE INFOCOM, New York, NY, Mar 1999.

[2] O. Gerstel, R. Ramaswami, and G. Sasaki, "Fault tolerant multiwavelength optical rings with limited wavelength conversion," IEEE

INFOCOM , Kobe, Japan, Apr 1997.

[3] E. Modiano and A. Narula-Tam, "Survivable routing of logical topologies in WDM networks", IEEE INFOCOM, Anchorage, AK, Apr 2001.

[4] O. Crochat and J. Le Boudec, "Design protection for WDM networks," IEEE JSAC, Vol. 16, No. 7, Sep 1998.

[5] D. Bertsimas and J. Tsitsiklis, Introduction to linear optimization, Athena Scientific, Belmont, MA, 1997. 